\title{
Frequency responses of age-structured populations: Pacific salmon as an example
}

\author{
Lee Worden $^{\mathrm{a}, \mathrm{b}, *}$, Louis W. Botsford ${ }^{\mathrm{c}}$, Alan Hastings ${ }^{\mathrm{b}}$, Matthew D. Holland ${ }^{\mathrm{c}}$ \\ ${ }^{a}$ Department of Biology, Theoretical Biology Lab, McMaster University, LS 3321280 Main \\ Street West, Hamilton, ON L8S 4 K1 Canada \\ ${ }^{b}$ Department of Environmental Science 83 Policy, University of California, Davis, One \\ Shields Ave, Davis, California 95616 USA \\ ${ }^{c}$ Department of Wildlife, Fish, \& Conservation Biology, University of California, Davis, \\ One Shields Ave, Davis, California 95616 USA
}

\begin{abstract}
Increasing evidence of the effects of changing climate on physical ocean conditions and long-term changes in fish populations adds to the need to understand the effects of stochastic forcing on marine populations. Cohort resonance is of particular interest because it involves selective sensitivity to specific time scales of environmental variability, including that of mean age of reproduction, and, more importantly, very low frequencies (i.e., trends). We present an agestructured model for two Pacific salmon species with environmental variability in survival rate and in individual growth rate, hence spawning age distribution. We use computed frequency response curves and analysis of the linearized dynamics to obtain two main results. First, the frequency response of the population is affected by the life history stage at which variability affects the population; varying growth rate tends to excite periodic resonance in age structure, while varying survival tends to excite low-frequency fluctuation with more effect on total population size. Second, decreasing adult survival strengthens the cohort resonance effect at all frequencies, a finding that addresses the question of how fishing and climate change will interact.
\end{abstract}

\footnotetext{
* Corresponding author

Email addresses: worden.lee@gmail.com (Lee Worden), lwbotsford@ucdavis.edu (Louis W. Botsford), amhastings@ucdavis .edu (Alan Hastings), mdholland@ucdavis.edu (Matthew D. Holland)
}

Preprint submitted to Elsevier

November 9, 2018 
Keywords: Structured populations, Cohort resonance, Salmon, Variability

2

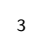

4

\section{Introduction}

In population theory, interest is increasing in the complex ways in which age-structured, density-dependent populations respond selectively to different time scales (or frequencies) of variability in the environment. These questions dovetail with the increasing practical need to understand how populations will respond to potential changes in life history parameters and the time scales of environmental variability due to climate change and increasing pressure on natural resources such as fisheries. There is a growing awareness that model population responses can appear to filter certain frequencies in environmental variability (Greenman and Benton, 2005). Furthermore, these effects can serve to amplify variability by exciting modes of population behavior that without environmental variability would be locally stable (Greenman and Benton, 2003). One example of such behavior, cohort resonance, is closely identified with life history characteristics in that it features cycles of period equal to the dominant age of reproduction. (Biørnstad et al., 1999).

There is particular interest in the dynamic responses of the marine fish targeted by global fisheries as they are subject to a dramatic artificial change in a life history parameter, adult survival, and there are indications that the dominant time scales of environmental variables such as ENSO that affect fish populations may change with a changing climate (Timmermann et al., 1999). Empirical observations and models indicate that variability in fish populations increases with increased fishing (Hsieh et al., 2006; Anderson et al., 2008). The cohort resonance phenomenon in fish populations presents particular problems for climate change because it enhances sensitivity to very slow signals (trends) in addition to those at the period of dominant age of reproduction (Biørnstad et al., 2004). Observations of such effects could be confounded with potential slow changes in the environment, and thus make it difficult to differentiate between them. 
Within the general concern for the combined effects of fishing and climate change on marine ecosystems (Perry et al., 2010), there is a particular interest in the effects of the marine environment and fishing on population dynamics of Pacific salmon on annual to decadal time scales. Analyses of the influences of the ocean environment on Pacific salmon, whether statistical examination of covariability between population and environmental variables (e.g., Logerwell et al., 2003) or estimation of survivals to specific sizes and ages through analysis of coded wire tagging data (e.g., Coronado and Hilborn, 1998; Teo et al., 2009), commonly assume the variable ocean environment influences survival during the early ocean phase of this anadromous genus. In fewer cases environmental variability in the age of maturation has been the dependent population variable (e.g., Pyper et al., 1999).

These empirical findings raise questions regarding the relative roles of random variability in survival at various ages, and in the age distribution of reproduction, in salmon population dynamics. Random survival is typically assumed to influence abundance directly, but the effects of varying age of spawning are not as clear, and there is a need to understand differences in population response to these two sources of random variability.

Here we examine and compare the effects of environmental variability in survival and age of spawning on the magnitude and time scales of population variability. We are engaged in a study of the influence of the oceanographic environment on Pacific salmon, the GLOBEC North East Pacific program, part of the US Climate Change program. Earlier retrospective analyses indicated differences in the responses of the two California Current congeners, chinook salmon, Oncorhynchus tshawytscha, and coho salmon, O. kisutch, and hence we are interested in the population dynamic differences between them (Botsford and Lawrence, 2002). For instance, coho salmon collapsed synchronously along the coast between 1980 and 2000, and coho appear to have more high frequency variability than chinook in their catch record. These species differ in their age distribution of spawning and in other ways (Botsford et al., 2005). We have explored some of the differences in probabilities of extinction of populations 
with these spawning age distributions in response to time-varying marine survival (Hill et al., 2003; Botsford et al., 2005). In salmon, differences in maturity schedule are due to differences in size distribution (and therefore prior growth rate) (Young, 1999; Vollestad et al., 2004). Similar effects occur in other nonsalmonid species (Day and Rowe, 2002). To avoid confusion, we note that the mechanisms studied here are neither (1) the indirect effect on survival of varying development rate due to consequent variation in time spent at higher mortality (e.g., Moloney et al., 1994), nor (2) the interaction between age structure and over-compensatory density-dependent recruitment that causes cycles of period roughly twice the generation time (Ricker, 1954; Botsford, 1997).

In the models used here oscillations with period $2 T$ do not arise (where $T$ is generation time or age of dominant effect on recruitment), primarily because of the form of stock-recruitment function we use, but the cyclic mode with period $T$ turns out to play a central role in our investigation. Recently Myers et al. (1998) explored stochastic forcing of a cyclic mode of variability of period $T$ as a potential cause of observed cycles in sockeye salmon (Oncorhynchus nerka) in British Columbia, Canada. This mode is similar to the "echo effect" associated with linear age-structured models of semelparous species (Sykes, 1969). Myers et al. noted that while this mode would not be the dominant mode, it could appear clearly in solutions obtained through forcing by randomly varying survival. This was essentially cohort resonance, though they did not use that term.

The time scales (or frequency content) of the population response also depend on the mode of observation, e.g., whether the data in a time series are recruitment, abundance or catch (Botsford, 1986; Anderson et al., 2008). The nature of a catch time series depends on fishing mortality rate, with higher fishing rates leading to higher frequencies of variability (Botsford, 1986; Hsieh et al., 2006). Differences between the spectra of recruitment and abundance have been illustrated by Biørnstad et al. (2004) for Atlantic cod (Gadus morhua). The most commonly available measurement of salmon populations is annual catch, either in numbers or biomass, and occasionally the age distribution of catch is determined. Frequently catch can be assumed to be individuals who if not 
caught would be spawning that year because they are near the mouth of (or in) the spawning river. In some streams, spawning escapement is also estimated, and sometimes the age composition of spawners is also estimated. Catch and escapement can be summed to obtain the total abundance of potential spawners in a year. Total population abundance at time $t$ cannot be observed directly, but estimates can be obtained through cohort reconstruction from several years of age-specific data from catch and escapement.

Here we explore several aspects of the spectral response of marine fish population dynamics, using parameter values representative of two species of Pacific salmon over a range of survivals as examples. Our practical interests are in the combined effects of fishing and climate change, so we focus on effects of long-term changes in survival on the population response to various time scales of environmental variability. Note, however, that long-term declines in survival can also be caused by the climate, independent of the fishery, as occurred in California Current coho salmon between 1980 and 2000 (Botsford et al., 2005). We also explore the differences in responses to environmental forcing at different points in the life history (i.e. development rate vs. survival), and spectral differences between various kinds of observations (e.g., observations of recruitment vs. observations of total abundance). We present results in terms of the changing spectral responses, and relate the spectral response to the eigenvectors and eigenspace structure of the linearized model.

\section{Model Formulation}

To investigate these questions, we introduce a density-dependent, stochastic age-structured model which we can use to compare the effects of temporal variability in the timing of reproduction and annual survivorship of salmon. Let $n$ be the maximum spawning age in years, and let $\vec{x}(t)=\left(x_{1}(t), \ldots, x_{n}(t)\right)^{\mathrm{T}}$ be 
the age-structured population vector at year $t$. Then our model is

$$
\vec{x}(t)=F(\vec{x}(t-1), t)=\left(\begin{array}{c}
R(P(t)) \\
s(t) x_{1}(t-1) \\
s(t) x_{2}(t-1) \\
\vdots \\
s(t) x_{n-3}(t-1) \\
\left(1-\delta_{e}(t)\right) s(t) x_{n-2}(t-1) \\
\delta_{l}(t) s(t) x_{n-1}(t-1)
\end{array}\right)
$$

where $P(t)=\left(\delta_{e}(t) x_{n-2}(t-1)+\left(1-\delta_{l}(t)\right) x_{n-1}(t-1)+x_{n}(t-1)\right)$ is the number of fish returning to spawn in year $t$, and

$$
R(P(t))=\frac{\alpha P(t)}{1+\beta P(t)}
$$

is a Beverton-Holt density dependent recruitment term (Beverton and Holt, 1957). Thus the recruitment $R(P(t))$ represents the number of outmigrants (smolts) leaving a spawning stream in year $t$, resulting from egg production by individuals of various spawning ages. We have assumed that migration from freshwater to the marine environment occurs in the first year, and that there is no difference in fecundity between the different ages of spawning. Parameters $\alpha$ and $\beta$ characterize the density-dependent reproductive phase: $\alpha$ is the density-independent per-capita growth rate when the population is very small, and $\frac{\alpha}{\beta}$ is the maximum total number of offspring in the population. Timing of reproduction is controlled by the remaining two parameters, $\delta_{e}(t)$ and $\delta_{l}(t)$ : most individuals spawn at age $n-1$; a proportion $\delta_{e}(t)$ of those surviving to age $n-2$ spawn at that age in year $t-1$; and a proportion $\delta_{l}(t)$ of age $n-1$ fish in year $t$ postpone spawning until year $t+1$, when they are age $n$. Annual survival $s(t)$, as written here, affects all age classes in year $t$. However, we also explore the possibility that the dominant variability in ocean survival occurs during the period immediately following ocean entry. This is a challenging stage for juvenile salmon, as they are completing the transition from freshwater to the marine environment, and they are dependent on the highly variable food production in the coastal ocean. 
We are interested in understanding the time scales of variation in the solutions to the model (equation 1) in response to fluctuations in annual survivorship and timing of spawning. We do this by studying three cases: the case of fluctuating survival $s(t)$ at all ages, the case in which survival varies but only at the age of entry into the ocean, and the case in which fluctuating mean age of spawning $a(t)$ produces yearly changes in $\delta_{e}(t)$ and $\delta_{l}(t)$. In all cases, the environmental fluctuation is a Gaussian white-noise signal, $\xi(t) \in \mathbb{R}$ with $\mathrm{E}(\xi(t))=0$. To model fluctuating survival we use $s(t)=s^{\circ}+\xi(t)$, where $s^{\circ}$ is the unperturbed, constant survival. In the simulation the distribution of $s(t)$ is truncated to ensure that $0 \leq s(t) \leq 1$. To model fluctuating age of spawning, we suppose that individuals' ages of spawning are chosen from a normal distribution whose mean is the central age of spawning $a(t)$ in year $t$ (Fig. 1), specifically

$$
p(a-a(t))=\frac{1}{\sqrt{2 \pi} \sigma} e^{-\left(\frac{a-a(t)}{\sqrt{2} \sigma}\right)^{2}} .
$$

We approximate early and late spawning by having all early spawners spawn at age $n-2$ and all late spawners spawn at age $n$, so that

$$
\delta_{e}(t)=\int_{-\infty}^{n-1.5} p(a-a(t)) \mathrm{d} a
$$

and

$$
\delta_{l}(t)=\int_{n-0.5}^{\infty} p(a-a(t-1)) \mathrm{d} a .
$$

From the point of view of a cohort, the portions of that cohort that will spawn early and late, i.e., $\delta_{e}$ and $\delta_{l}$, are set by the value of $a(t)$ in the year that that cohort transitions from age $n-2$ to age $n-1$. That is, $\delta_{e}(t)$ is a function of the central spawning age in year $t$, whereas $\delta_{l}(t)$, which has its effect a year after it is determined, is a function of the central spawning age in year $t-1$. We model the fluctuating central age of spawning as $a(t)=a^{\circ}+\xi(t)$.

To compare the dynamics of the two salmon species along the west coast of the contiguous U.S., we have used parameter values that approximate known or likely values for the populations of coho and chinook salmon. Survival rates in the ocean are reasonably well known, and we consider three cases: a "typical" value of $0.85 \mathrm{yr}^{-1}$ (Bradford, 1995), a "small" value of $0.28 \mathrm{yr}^{-1}$, 


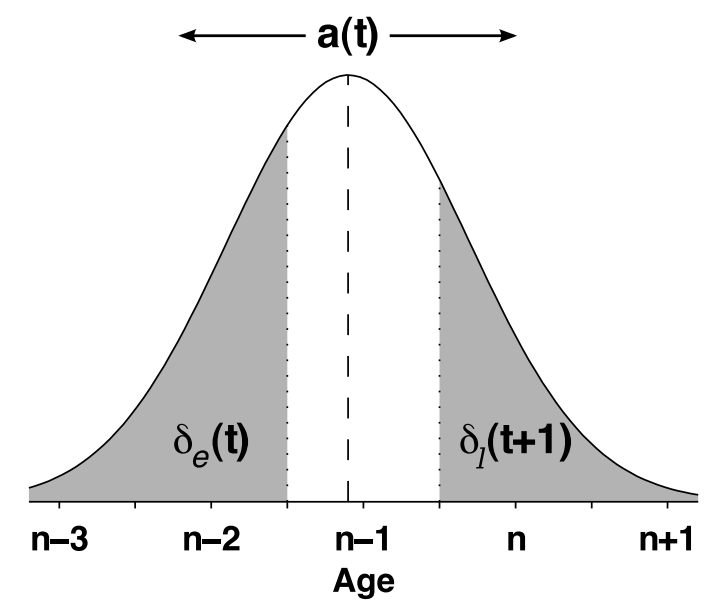

Figure 1: An example illustrating how the fraction of early and late spawners is modeled. The central spawning age $a(t)$ varies from year to year. Here we picture a normal distribution of potential spawning age with mean $a(t)=n-1.1$ and $\sigma=0.8$. The parameters representing the proportions of early spawners $\left(\delta_{e}\right)$ in year $t$ and late spawners $\left(\delta_{l}\right)$ in year $t+1$ are the integrals of this function over $(-\infty, n-1.5]$ and $[n-0.5, \infty)$, respectively.

and a "very small" value of $0.2 \mathrm{yr}^{-1}$, estimated from more recent observations of coho salmon. Since the "very small" value is not sustainable in the chinook model, that case is not considered. The distributions of values of $\alpha$ and $\beta$ (equation 2) have been estimated for coho salmon, but not for chinook salmon (Barrowman et al., 2003), and we use the modes from those distributions ( $\alpha=60$ and $\beta=0.00017$ ). In chinook salmon populations in the California Current, individuals typically spawn primarily at a single dominant age, with less spawning at adjacent ages. The dominant age of spawning increases with latitude (Hill et al., 2003). Here we chose dominant spawning at age 4, i.e., $n=5$ and $a^{\circ}=4$. Precocious spawning in chinook salmon ranges from $0-$ 10 percent in males and from 0-3 percent in females (Healey, 1991). We use $\sigma=0.4$, which makes $\delta_{e}=\delta_{l} \approx 0.1$. Coho salmon in the California Current spawn predominantly at age 3 with substantial precocious spawning at age 2 and minimal spawning at age 4. Precocious spawning in coho salmon consists almost completely of males, is more variable than in chinook salmon, and can be as high as 30 percent in natural wild populations (Sandercock, 1991). It is not 
variation in survival, and 0.2 for variation in mean spawning age.

possible to observe the effects of precocious spawning by males on reproduction directly, but for coho salmon, a modification of genetic methods for estimating effective population size indicated the effective proportion of 2 -year-olds to be 35 percent in two naturally spawning populations (Doornik et al., 2002). For coho salmon we chose $n=4, a^{\circ}=2.75$ and $\sigma=0.2$, which makes $\delta_{e} \approx 0.1$ and $\delta_{l} \approx 0.0001$. The standard deviation of the stochastic variation, $\sigma_{E}$, is 0.085 for

Table 1: Model parameters and unperturbed values.

\begin{tabular}{|c|l|c|c|}
\hline Parameter & Description & Coho Value & Chinook Value \\
\hline$s^{\circ}$ & annual survival rate, all ages & $0.85,0.28,0.2$ & $0.85,0.28$ \\
\hline$\delta_{e}$ & $\begin{array}{l}\text { proportion of age } n-2 \text { fish spawn- } \\
\text { ing }\end{array}$ & 0.1056 & 0.1056 \\
\hline$\delta_{l}$ & $\begin{array}{l}\text { proportion of age } n-1 \text { fish delay- } \\
\text { ing spawning to age } n\end{array}$ & $8.84 \times 10^{-5}$ & 0.1056 \\
\hline$\alpha$ & $\begin{array}{l}\text { slope at origin of Beverton-Holt } \\
\text { stock-recruitment curve }\end{array}$ & 60 & 60 \\
\hline$\beta$ & $\begin{array}{l}\text { saturation parameter of } \\
\text { Beverton-Holt stock-recruitment } \\
\text { curve }\end{array}$ & $1.7 \times 10^{-4}$ & $1.7 \times 10^{-4}$ \\
\hline$a^{\circ}$ & $\begin{array}{l}\text { central age of spawning } \\
\text { standard deviation of spawning } \\
\text { age distribution }\end{array}$ & 0.2 & 0.4 \\
\hline$\sigma$ & $\begin{array}{l}\text { standard deviation of environ- } \\
\text { mental forcing signal }\end{array}$ & 0.085 (survival) & 0.085 (survival) \\
\hline$\sigma_{E}$ & 0.2 (spawning age) & 0.2 (spawning age) \\
\hline
\end{tabular}

187

\section{Model Analysis}

In each of the model cases, the age vector $\vec{x}$ converges to a neighborhood of a single point, and fluctuates in that neighborhood in response to the fluctuating life-history parameters. To find the center point, we consider the deterministic system defined by equation 1 with $s, \delta_{e}$, and $\delta_{l}$ all fixed at their unperturbed values. This system has a unique positive fixed point that is globally attracting 
for positive trajectories:

$$
\tilde{\vec{x}}=\left(\begin{array}{c}
1 \\
s \\
s^{2} \\
\vdots \\
s^{n-3} \\
\left(1-\delta_{e}\right) s^{n-2} \\
\left(1-\delta_{e}\right) \delta_{l} s^{n-1}
\end{array}\right) \frac{\alpha-c}{\beta},
$$

where $1 / c=\delta_{e} s^{n-3}+\left(1-\delta_{e}\right)\left(1-\delta_{l}\right) s^{n-2}+\left(1-\delta_{e}\right) \delta_{l} s^{n-1}$ is the amount of spawning in the lifetime of the average smolt.

As we will see, we can understand a great deal about the population dynamics by looking at the linearized dynamics at this fixed point of the deterministic dynamics. We linearize the effect of the stochastic noise term $\xi(t)$ as well as the age-structured population vector $\vec{x}(t)$ :

$$
\vec{y}(t) \approx \mathbf{J} \vec{y}(t-1)+\sum_{l=0}^{L} \vec{H}(l) \xi(t-l),
$$

where $\vec{y}(t)=\vec{x}(t)-\tilde{\vec{x}}(t)$ is the deviation from the fixed point, $\mathbf{J}$ is the Jacobian matrix of the dynamics at the fixed point, and linearizing in the noise term gives a sequence of "forcing vectors" $\vec{H}$ that summarizes how the noise enters into the dynamics with various time lags (see the Appendix for details).

At the fixed point, the Jacobian matrix is

$$
\mathbf{J}=\left(\begin{array}{cccccc}
0 & \cdots & 0 & \delta_{e} \frac{c^{2}}{\alpha} & \left(1-\delta_{l}\right) \frac{c^{2}}{\alpha} & \frac{c^{2}}{\alpha} \\
s & & & & & 0 \\
& \ddots & & & & \\
& & s & & & \vdots \\
& & & \left(1-\delta_{e}\right) s & & \\
& & & & \delta_{l} s & 0
\end{array}\right),
$$

where all entries left blank are zero. 
A linear system like this one decomposes naturally into independent subsystems located in linearly independent one- and two-dimensional subspaces, each characterized by a real eigenvalue or a complex pair of eigenvalues (Hirsch and Smale, 1974). The right eigenvectors of the Jacobian matrix are the basis vectors for each of these subspaces of the dynamics. The complex conjugate eigenvalues of the Jacobian predict the resonant frequencies of the population's response to noise. In the time-varying-survival cases, we only need a single forcing vector $\vec{H}$, because the state of the environment in year $t, \xi(t)$, only affects survival in year $t$; but in the varying-age-structure case, because conditions in year $t$ affect the number of early returns $\delta_{e}$ in year $t$ and the number of late returns $\delta_{l}$ in year $t+1$, we have to include two forcing vectors to describe the effects with and without one year's time lag.

In the case of fluctuating survival at all ages, the forcing is captured by the vector $\vec{H}_{s}(0)$, with

$$
\vec{H}_{s}(0)=\left(\frac{\partial F_{i}}{\partial \xi(t)}\right)=\left(\begin{array}{c}
0 \\
\tilde{x}_{1} \\
\tilde{x}_{2} \\
\tilde{x}_{3} \\
\vdots \\
\tilde{x}_{n-3} \\
\left(1-\delta_{e}\right) \tilde{x}_{n-2} \\
\delta_{l} \tilde{x}_{n-1}
\end{array}\right)=\left(\begin{array}{c}
0 \\
1 \\
s^{\circ} \\
\left(s^{\circ}\right)^{2} \\
\vdots \\
\left(s^{\circ}\right)^{n-4} \\
\left(1-\delta_{e}\right)\left(s^{\circ}\right)^{n-3} \\
\left(1-\delta_{e}\right) \delta_{l}\left(s^{\circ}\right)^{n-2}
\end{array}\right) \frac{\alpha-c}{\beta} .
$$

We note that when survival is forced additively, as we have done here, the deterministic system described above is not exactly the mean of the stochastic system. A stochastic system with $s(t)=s^{\circ} e^{\xi(t)}$ would have the deterministic system as its mean, but in the limit of small noise, these representations have identically-shaped frequency responses that merely differ by a factor of $s$. We chose additive noise for convenience.

For forcing of survival at ocean entry, only survival to age 2 is subject to 


$$
\vec{H}_{s_{e}}(0)=\left(\begin{array}{c}
0 \\
\frac{\alpha-c}{\beta} \\
0 \\
\vdots \\
0
\end{array}\right) .
$$

229 For time-varying ages of maturation, we have lag 0 effects from early spawn230 ing

$$
\vec{H}_{a}(0)=\left(\frac{\partial F_{i}}{\partial \xi(t)}\right)=\left(\frac{\partial F_{i}}{\partial \delta_{e}} \frac{\partial \delta_{e}}{\partial \xi(t)}\right)
$$

231 and lag 1 effects from late spawning

$$
\vec{H}_{a}(1)=\left(\frac{\partial F_{i}}{\partial \xi(t-1)}\right)=\left(\frac{\partial F}{\partial \delta_{l}} \frac{\partial \delta_{l}}{\partial \xi(t-1)}\right) .
$$

${ }_{232}$ Since $\frac{\partial a(t)}{\partial \xi(t)}=1$ we have

$$
\frac{\partial \delta_{e}(t)}{\partial \xi(t)}=\int_{-\infty}^{n-1.5}-p^{\prime}(a-a(t)) \mathrm{d} a=-p(n-1.5-a(t))
$$

233 and

$$
\frac{\partial \delta_{l}(t)}{\partial \xi(t-1)}=\int_{n-0.5}^{\infty}-p^{\prime}(a-a(t-1)) \mathrm{d} a=p(n-0.5-a(t-1)) .
$$

${ }_{234}$ The vector derivatives we need are

$$
\left(\frac{\partial F_{i}}{\partial \delta_{e}}\right)=\left(\begin{array}{c}
s^{n-3} \frac{c^{2}}{\alpha} \\
0 \\
\vdots \\
0 \\
-s^{n-2} \\
0
\end{array}\right) \frac{\alpha-c}{\beta}
$$

235 and

$$
\left(\frac{\partial F_{i}}{\partial \delta_{l}}\right)=\left(\begin{array}{c}
-\left(1-\delta_{e}^{\circ}\right) s^{n-2} \frac{c^{2}}{\alpha} \\
0 \\
\vdots \\
0 \\
\left(1-\delta_{e}^{\circ}\right) s^{n-1}
\end{array}\right) \frac{\alpha-c}{\beta}
$$


where $\delta_{e}^{\circ}$ refers to the unperturbed value of $\delta_{e}$. Substituting (13) and (15) into (11) yields the forcing at lag 0 ,

$$
\vec{H}_{a}(0)=\left(\begin{array}{c}
-p\left(n-1.5-a^{\circ}\right) s^{n-3} \frac{c^{2}}{\alpha} \\
0 \\
\vdots \\
0 \\
p\left(n-1.5-a^{\circ}\right) s^{n-2} \\
0
\end{array}\right) \frac{\alpha-c}{\beta} .
$$

Substituting (14) and (16) into (12) gives the forcing at lag 1 ,

$$
\vec{H}_{a}(1)=\left(\begin{array}{c}
-p\left(n-0.5-a^{\circ}\right)\left(1-\delta_{e}^{\circ}\right) s^{n-2} \frac{c^{2}}{\alpha} \\
0 \\
\vdots \\
0 \\
p\left(n-0.5-a^{\circ}\right)\left(1-\delta_{e}^{\circ}\right) s^{n-1}
\end{array}\right) \frac{\alpha-c}{\beta} .
$$

\section{Results}

\subsection{Dynamics near the fixed point}

Each of these models without random fluctuation has a unique positive fixed point (equation (6)). The condition for a fixed point can be illustrated in terms of the stock-recruitment function and a straight line through the origin with slope equal to the inverse of mean lifetime egg production, as shown in Fig. 2 (Sissenwine and Shepherd, 1987). The fixed point lies at the intersection of these, allowing clear interpretation of the effects of long-term changes in survival. As mean ocean survival declines, equilibrium egg production declines, and eventually recruitment declines. For the very low survival rate for chinook salmon, the fixed point is zero indicating survival is not adequate for persistence. Because the parameters for the stock-recruitment relationship and the estimated survivals are for coho salmon, this has no implications for real chinook salmon populations. 


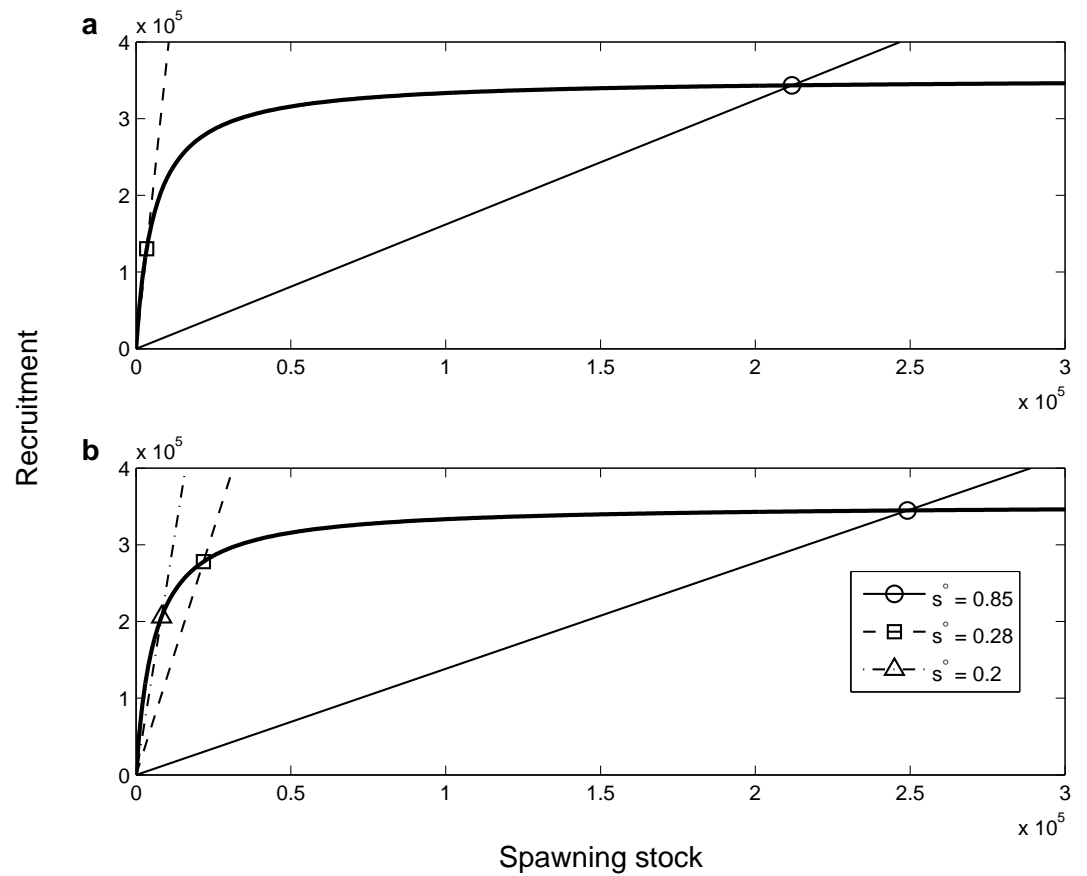

Figure 2: Graphical interpretation of the equilibrium recruitment as the intersection of the smolt-adult curve and a line through the origin with slope $1 /$ (lifetime reproduction) for (a) chinook and (b) coho salmon. 

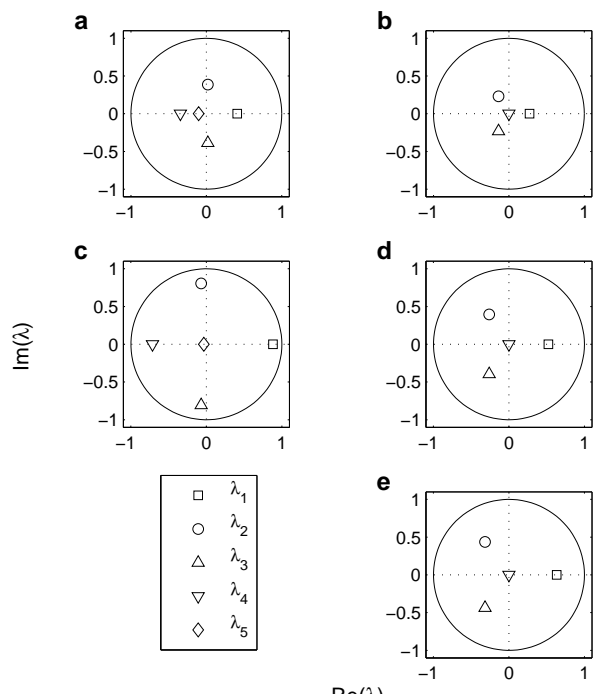

Figure 3: Eigenvalues of linearization matrix $\mathbf{J}$ in chinook and coho models for typical survival: (a) chinook, $s=0.85 \mathrm{yr}^{-1}$, (b) coho, $s=0.85 \mathrm{yr}^{-1}$; small survival: (c) chinook, $s=0.28 \mathrm{yr}^{-1}$, (d) coho, $s=0.28 \mathrm{yr}^{-1}$; and very small survival: (e) coho, $s=0.2 \mathrm{yr}^{-1}$.

Plots of the eigenvalues of the linearization at the fixed point of each of these models indicate the modes of variability, and how they change with survival (Figure 3). Note that the chinook model has 5 eigenvalues, while the coho model has 4 eigenvalues because those are the maximum ages in each model population. In both the chinook and coho models, the linearized population dynamics respond most strongly to forcing in the one-dimensional mode corresponding to the positive real eigenvalue, $\lambda_{1}$ (the dominant mode of variability). Motion in this mode occurs without oscillation, that is, at low frequencies. The next strongest mode is a resonance at period $n-1$ or a little less (determined by the eigenvalues $\lambda_{2}$ and $\lambda_{3}$, whose angle from the positive axis is approximately $\pm \frac{2 \pi}{n-1}$ ), indicating oscillations with a period equal to one generation time. In the chinook case (Figures 3a,c) there is also a strong resonance at period two, corresponding to a negative eigenvalue, and in all cases there is a weakly resonating negative eigenvalue as well.

In both the chinook and the coho cases, the effect of decreasing the mean survival is to move all eigenvalues outward toward the unit circle, increasing the 
return time of all subsystems of interest and thereby increasing the expected magnitude of the cumulative response to variation over time. This effect is especially strong in the chinook model for "small" $s$ (Fig. 3r). This occurs because the rate of change of recruitment with adult stock increases substantially as the survival declines (Fig. 2). Therefore, low equilibrium abundances in Figure 2 correspond to large magnitude eigenvalues (and, thus, large responses to variation)in Figure 3 .

The spread in ages of spawning has a relatively small specific influence on the locations of the eigenvalues. When $\sigma=0$, all fish spawn at age $n-1$, and the eigenvalues are roots of a simple characteristic polynomial, $P(\lambda)=$ $\lambda\left(\lambda^{n-1}-s^{n-2} \frac{c^{2}}{\alpha}\right)$. There is one zero root and the others are equal in size and evenly spaced around zero (not shown). As $\sigma$ increases, the dominant, positive real eigenvalue moves outward on the negative real axis, the others move inward slightly and the complex eigenvalues rotate slightly, their polar angles becoming slightly smaller in the chinook case and slightly larger in the coho case. In all cases other than $\sigma=0$, the positive eigenvalue is largest in magnitude (consistent with the Perron-Frobenius theorem, since the entries of $\mathbf{J}$ are nonnegative). For our purposes, we conclude that the spread in spawning age has very little impact on the model dynamics (too slight to justify an illustration, in fact), and so our models do not indicate that it is an important difference between chinook and coho population dynamics.

\subsection{Mechanism of environmental forcing}

To illustrate the difference between population responses to different mechanisms of environmental forcing we compare the cases with (1) variability in survival in each ocean year, (2) variability in survival at the age of ocean entry only and (3) variability in the spawning age distribution. Random variability in survival appears to preferentially excite the geometrically decaying mode, while variability in the spawning age preferentially excites the cyclic mode. This conclusion holds for all five of our model cases: the resonant frequency is about $0.33 \mathrm{yr}^{-1}$ for the coho salmon, whose dominant age of spawning is three years, 
and about $0.25 \mathrm{yr}^{-1}$ for the chinook salmon, whose dominant age of spawning is four years (not shown here, see next example). The one exception is that for the chinook model with $s^{\circ}=0.28 \mathrm{yr}^{-1}$, there is a strong low-frequency component with variation in spawning age as well as the period-4 component.

We illustrate this general result with the case for coho salmon with $s^{\circ}=$ $0.28 \mathrm{yr}^{-1}$ by comparing the frequency responses to survival varying at all ages, for survival varying only at age 1 , the presumed age of ocean entry, and for variability in spawning age, then presenting examples of time series for each case. Both frequency responses to environmental variability in survival (Fig. 4a) decline from very low frequency, leveling off slightly at a frequency just below that corresponding to period 3 (the dominant age of spawning for coho salmon), then decline for higher frequency. The simulations indicate up to approximately 20 percent greater variability than the analytical model in both survival cases. The case with variable ocean survival at all ages (Fig. 4b) is skewed more toward variability at low frequencies. The frequency response to environmental variability in the spawning age distribution increases from low frequency to a resonance at a frequency slightly greater than that corresponding to period three, the dominant age of spawning, consistent with cohort resonance.

The time series for each case (Fig. 5]) indicate a visually discernable difference between populations driven by environmental variability in survival and environmental variability in spawning age distribution. The population with environmental forcing of spawning age (Fig. 5r) has clear indications of cohort resonant behavior near period 2 yr to $4 \mathrm{yr}$, while the populations with environmental forcing of survival tend toward 5-10 yr fluctuations with little variability. The difference in the magnitude of variability between the series with varying survival is as expected from the difference in area under the two thin lines in Fig. 4 .

\subsection{Population observation}

To determine the effects of the type of observation on the time scales of variability we compared the results of observing recruitment to the results of 
a

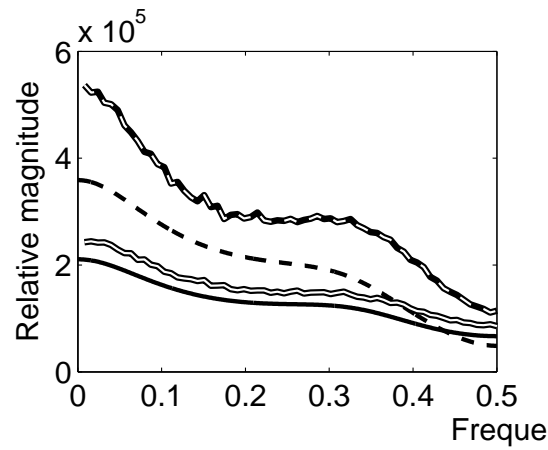

b

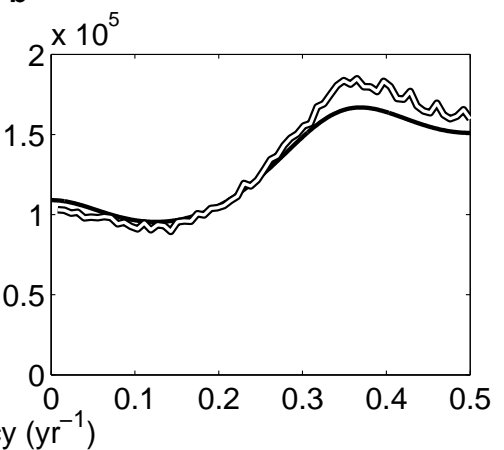

Figure 4: Magnitude of frequency response of recruitment for the linearized model (thin lines) and nonlinear simulations (bordered lines) for environmental forcing of (a) early ocean survival rate (solid lines) and ocean survival at all ages (dashed lines), and (b) spawning age distribution for coho salmon with $s^{\circ}=0.28 \mathrm{yr}^{-1}$. See the Appendix (equation A.10) for derivation of the linearized frequency reponse. Nonlinear frequency response curves are estimated by calculating the average magnitude (the square root of mean power at each frequency) of the fast Fourier transforms of 1000 simulation time series of length 128, and scaling the result by $\sqrt{128} \sigma_{E}$, to obtain the same units as the analytically-derived transfer function.

observing total abundance for each case. Generally, they all exhibited relatively more 2-to-5-year oscillation in the recruitment and more low-frequency fluctuation in the total abundance.

To illustrate the difference between different types of population observations, we show the results of observing recruitment with the results of observing total abundance, for chinook salmon with typical survival $\left(s=0.85 \mathrm{yr}^{-1}\right)$ and environmental variability in the spawning age distribution (Fig. 6). The spectral response of recruitment increases from low frequency to a peak at about $0.25 \mathrm{yr}^{-1}$, the frequency expected for chinook salmon with dominant age of reproduction at $4 \mathrm{yr}$, then declines. The spectral response of total abundance, the sum of several cohorts, declines monotonically from low frequency, showing only a hint of the resonance present in recruitment.

The time series of these two cases (Fig. 7) reflect these characteristics. The time series from the population with observations of recruitment of a chinook salmon population with environmental forcing of spawning age appears to have a preponderance of variability on time scales of $2 \mathrm{yr}$ to $5 \mathrm{yr}$, while the time 
a $\times 10^{5}$
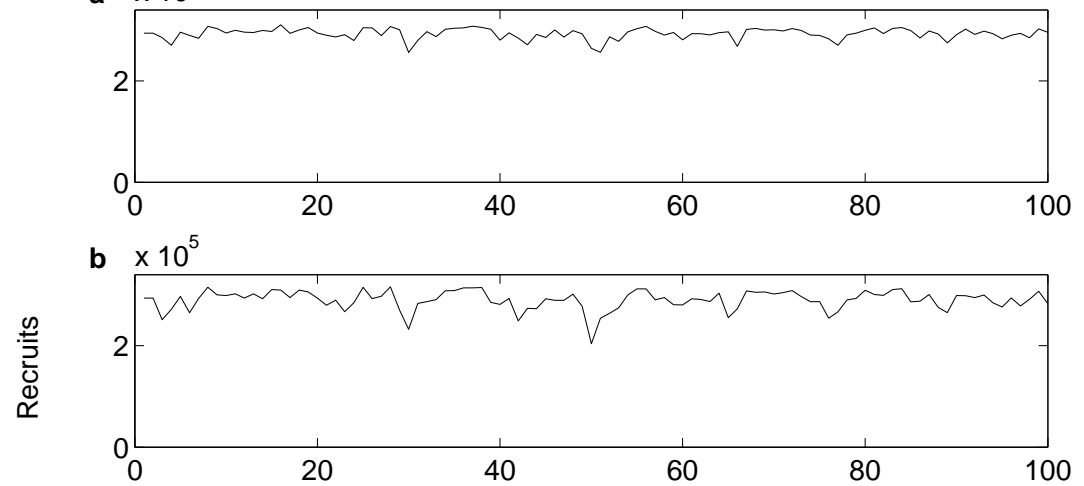

c $\times 10^{5}$

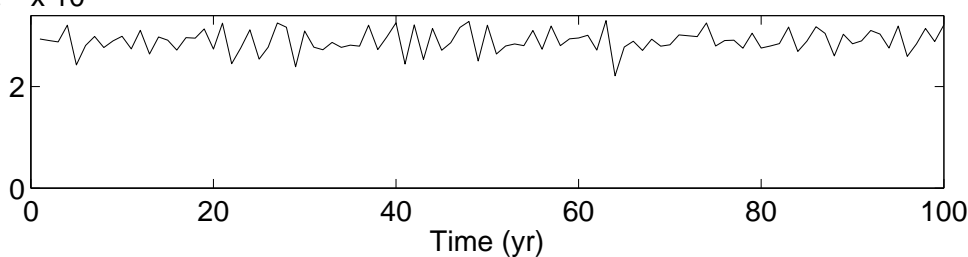

Figure 5: Time series of recruitment in simulations with environmental forcing of (a) early ocean survival $\left(\sigma_{E}=0.085\right)$, (b) ocean survival at all ages $\left(\sigma_{E}=0.085\right)$, and (b) spawning age distribution $\left(\sigma_{E}=0.2\right)$ for coho with $s^{\circ}=0.28 \mathrm{yr}^{-1}$. Identical sequences of standard normal random variables were used as the forcing signal in order to allow a comparison of filtering by different demographic mechanisms.

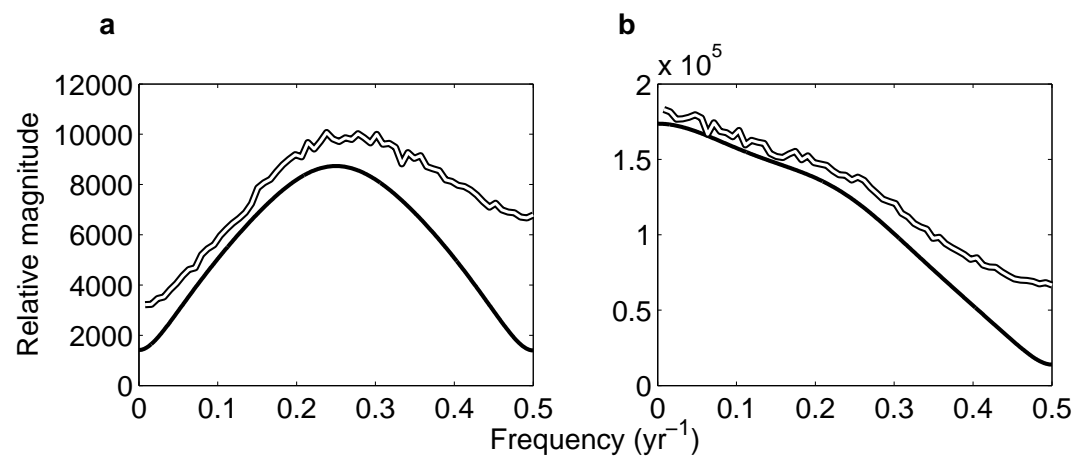

Figure 6: Magnitude of frequency response of (a) recruitment and (b) total abundance for the linearized model (black line) and nonlinear simulations (bordered line; 1000 simulations of length 128) for environmental forcing of spawning age distribution for chinook salmon with $s=0.85 \mathrm{yr}^{-1}$. 

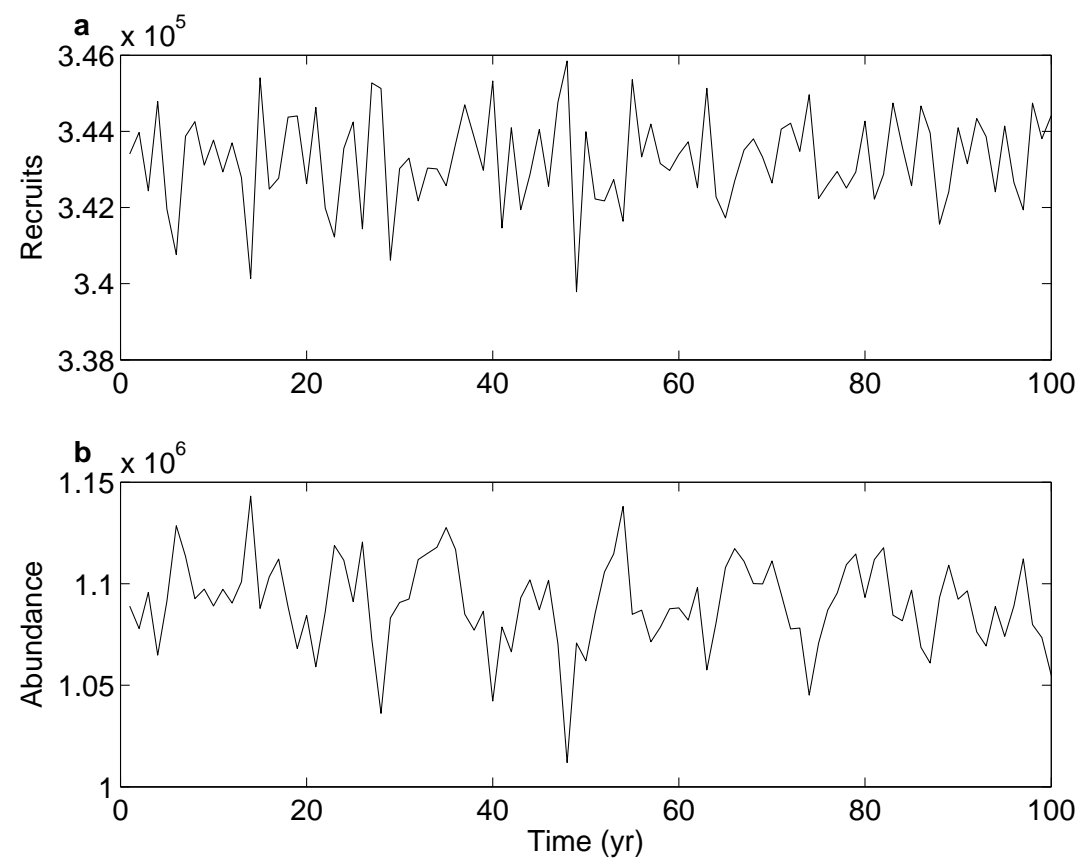

Figure 7: Time series of (a) recruitment and (b) total abundance from a single simulation with environmental forcing of spawning age distribution $\left(\sigma_{E}=0.2\right)$ for chinook salmon with $s=0.85 \mathrm{yr}^{-1}$.

series from the observation of abundance appears to be a smoothed version of that.

\subsection{Long-term mean survival}

To illustrate the differences between populations operating at different levels of long-term survival we compared the coho salmon recruitment from a model with environmental variability in spawning age distribution with each of the three survival levels, typical, small and very small (Fig. 8). The shift to lower constant survivals could result from fishing or a shift in climate. The frequency responses with these three survivals have similar shapes but differ substantially in scale. The resonance at period just less than 3 years is dominant because the variability is in spawning age distribution. Note the greater increase in sensitivity to low frequencies as the survival decreases to its lowest level. 


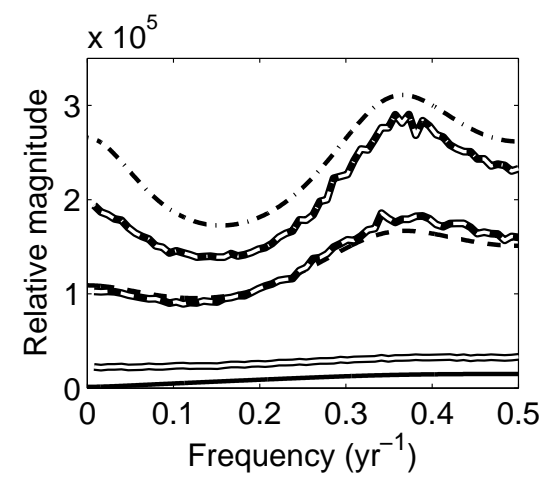

Figure 8: Magnitude of frequency response of recruitment for the linearized model (thin lines) and nonlinear simulations (bordered lines; 1000 simulations of length 128 with $\sigma_{E}=0.2$ ) for environmental forcing of spawning age distribution for coho with $s=0.85 \mathrm{yr}^{-1}$ (solid lines), $0.28 \mathrm{yr}^{-1}$ (dashed lines), and $0.2 \mathrm{yr}^{-1}$ (dash-dot lines).

373 Biørnstad et al., 2004).

\section{Discussion}

The time series of these three cases (Fig. 9) appear to have similar frequency content, but different levels of variability as expected from Fig. 8. Importantly, they also underscore the fact that as survival declines variability increases as in Fig. 8, as the equilibrium recruitment declines.

These analyses provide an understanding of how various population characteristics shape the response of salmon populations to environmental variability on various time scales. Salmon population variability does not simply follow variability in the environment as is commonly assumed, but rather the observed response is shaped by three factors: (a) the life history point of impact of the environment, (b) how the population is censused, and (c) pre-conditioning by long-term changes in survival. These considerations have important consequences for the management of salmon populations and the anticipation of the effects of large-scale environmental change. The dynamics of salmon responses to the environment are, of course, not unique, but rather are closely related to those of other higher trophic level, age-structured species (McCann et al., 2003; 


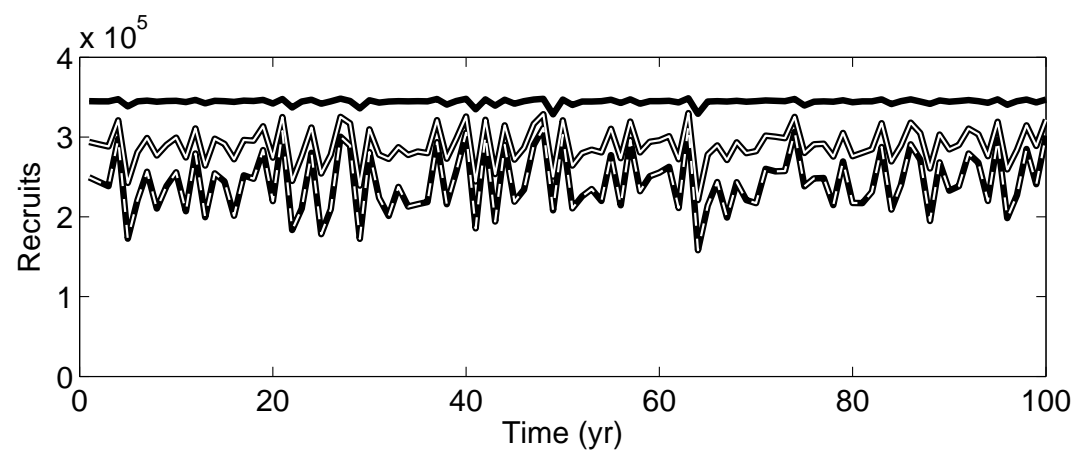

Figure 9: Time series of recruitment in simulations with environmental forcing of spawning age distribution $\left(\sigma_{E}=0.2\right)$ for coho with $s=0.85 \mathrm{yr}^{-1}$ (solid black), $0.28 \mathrm{yr}^{-1}$ (white with black border), and $0.2 \mathrm{yr}^{-1}$ (dashed white on black). Identical sequences of standard normal random variables were used as the forcing signal in order to allow a comparison of filtering at different survival rates.

The importance of cohort resonance to climate change is enhanced by the finding that its effects intensify with decreasing survival. There is concern over the effects of climate change on fisheries, and how management should change to mitigate those changes (e.g., Perry et al., 2010). The basic resonance mode with period $T$ appears as the second and third eigenvalues in the linearized model, a conjugate pair. It is well known in linear age-structured models, as the echo effect (Sykes, 1969; Caswell, 2001), and was identified earlier as a potential cause of observed cycles in some salmon populations (Myers et al., 1998). However, increasing cohort resonance with fishing has not been mentioned as a factor in the variability in fished populations. Knowing that high levels of fishing and specific time scales of environmental variability can increase overall population variability will be valuable in formulating management policies to maintain sustainability. The additional variability increases the risk of population collapse in addition to that risk incurred by reducing mean abundance. In addition, knowing that fish populations could be more sensitive to some time scales than others will aid in the explanation of changes in fisheries' variability if the time scales of forcing change with climate. Potential intensification of cohort resonance through increased fishing also underscores the importance of Bjørnstad's 
(2004) warning regarding the low-frequency effect of cohort resonance, sensitivity to very low-frequency environmental variability. Fishing increases the chances that populations, even when driven by white noise in the environment, could generate very slowly changing signals that could be mistaken for the effect of a slowly changing climate. This hard-to-detect long-timescale change may present a third increase in threat when coupled with reduced abundance and increased variability due to fishing. Note that this increased sensitivity at low frequencies was seen in coho salmon (Fig. 8) and also in chinook salmon with $s=0.28 \mathrm{yr}^{-1}$ (as noted at the end of section 4.2).

Differences in the dynamic responses of populations to temporal variability at different points in the species's life history are not commonly considered. For salmon (and most other marine fish), while the effects of variability in survival are widely appreciated, the authors are unaware of any description of the effects of variability in spawning age distribution on population dynamics, such as on cohort resonance. Here we found that the different mechanisms involving variability in survival and variability in spawning age distribution preferentially excited different fundamental modes of variability; hence these differences are clearly important. In addition to variability in spawning age exciting a completely different mode than variability in survival, responses to varying survival at ocean entry and varying total survival also differed, with less sensitivity to low frequencies resulting from variability in early ocean survival than from variability in total survival. This further motivates efforts to discover the ages and locations at which variability in salmon marine survival occurs. This may be related to the difference in population persistence between these two survival mechanisms in earlier analysis (Botsford et al., 2005), where population variability was greater when it occurred at the age of return to the river for spawning, rather than the age of ocean entry of smolts. In that case this difference occurs because of the Law of Large Numbers, and the fact that the logarithm of the number of spawners is the sum of several random survivals when variability is at the age of entry, but only one random survival when it is at the age of return. In our models, varying age of spawning causes much more oscillation at the 
period of the generation length and less low-frequency fluctuation than varying survival does. This might be because changes in central spawning age have a double effect on recruitment, removing fish from one year's stock of potential spawners and adding them to the next or previous year's potential spawning stock. This perturbation has a direct effect on two cohorts' recruitment, which is echoed in subsequent generations when those cohorts spawn. Fluctuations in survival, on the other hand, whether they affect one cohort or many, cannot have this double effect, with the likely consequence that the period- $T$ echo effect is not as extreme.

The difference in frequency content between a catch (or abundance) series and a recruitment series was known previously (e.g., Botsford, 1986), but there has been renewed interest in it. That difference basically follows from the Law of Large Numbers and the fact that when recruitment is the source of temporal variability, abundance will have a lower coefficient of variation because it is the sum of several recruitments, and hence can be expected to be a smoothed version of the recruitment signal, i.e., to emphasize lower frequencies. As the value of constant harvest rate increases, the number of cohorts summed diminishes, and hence higher frequencies are observed.

The major difference between the population dynamics of coho salmon and chinook salmon revealed here is that they would be most sensitive to different time scales of environmental variability, as determined by the difference between the dominant age of spawning in each. Also, one explanation proposed for the fact that coho salmon declined near 1980, while chinook salmon did not, was that narrower width of the coho spawning age distribution. The effect of width of the spawning age distribution we determined here was not strong enough to support this hypothesis, leaving open the possibility that coho salmon were merely following a decline in ocean survival which did not decline for chinook salmon. These theoretical results are not definitive regarding specific stocks; rather they provide a context for further detailed investigations of the hundreds of coho and chinook salmon populations along the west coast of the U.S. As with other structured-population models (Nisbet and Gurney, 1982; Biørnstad et al., 
2004; Greenman and Benton, 2003), this model's response to environmental fluctuation is predicted well by the linearization at the deterministic model's fixed point. Like Greenman and Benton, we find amplification of noise as parameters vary - in our case, environmental noise is amplified more by the population dynamics when survival is reduced. In our case, however, this amplification is not associated with a nearby bifurcation, only with eigenvalues moving closer to the unit circle, a more general phenomenon.

As discussed above, we also observe major differences in frequency responses among different kinds of environmental influences and between different population measurements. The analysis in our Appendix explains these differences in terms of different geometric relationships between the forcing vectors, the eigenspaces corresponding to the eigenvalues of the linearization, and the measurements. The projection of the forcing vectors $\vec{H}$ into each dynamical subspace determines how strongly the environmental noise stimulates motion in that subsystem, and therefore how prominent resonance at that frequency is in the population dynamics. Similarly, different observations - total population and recruitment — include the different subsystems in different proportions, and so each mode of population behavior is more visible in some observations than others (see the Appendix for the mathematical treatment of these ideas). This analysis, together with the diversity in frequency responses we see in our models, points to the importance of understanding the relationship between the eigenspace structure of the linearization and the relative importances of the resonant frequencies associated with each eigenspace. As an analytical technique, this approach may be useful in multispecies ecological models as well as in structured population models.

The results obtained here provide the means both to begin to explain current differences in responses to the environment by the same species at different locations, and to project differences in future responses on the basis of projected changes in time scales of variability in the environment. Current differences in responses by populations of the same species are typically presumed to imply a difference in environmental forcing, but they may be due to differences in life 
histories or differences in preconditioning because they are fished at different intensities. Future changes in time scales of variability are expected on the basis of paleological records (e.g., past changes in the time scales of variability of El Niño events as observed in corals and other media, Jones et al., 2009) or predictions from global climate models (Timmermann et al., 1999).

\section{Acknowledgments}

This research is part of the GLOBEC Northeast Pacific program and was funded by National Science Foundation grants NSF OCE0003254 and NSF OCE0815293. Any opinions, findings, and conclusions or recommendations expressed in this material are those of the author(s) and do not necessarily reflect the views of the National Science Foundation.

Anderson, C., Hsieh, C., Sandin, S., Hewitt, R., Hollowed, A., Beddington, J., May, R., Sugihara, G., 2008. Why fishing magnifies fluctuations in fish abundance. Nature 452 (7189), 835-839.

Barrowman, N., Myers, R., Hilborn, R., Kehler, D., Field, C., 2003. The variability among populations of coho salmon in the maximum reproductive rates and depensation. Ecological Applications 13, 784-793.

Bernstein, D., 1992. Some open problems in matrix theory, arising in linear systems and control. Linear Algebra and its Applications (162-164), 409-432.

Beverton, R., Holt, S., 1957. On the dynamics of exploited fish populations. Fishery Investigations, Series II. Marine Fisheries, Great Britain Ministry of Agriculture, Fisheries and Food 19, 533.

Bjørnstad, O. N., Fromentin, J. M., Stenseth, N., Gjøsæter, J., 1999. Cycles and trends in cod populations. Proceedings of the National Academy of Sciences USA $96,5066-5071$.

Bjørnstad, O. N., Nisbet, R. M., Fromentin, J. M., 2004. Trends and cohort resonant effects in age-structured populations. Journal of Animal Ecology 73 (6), 1157-1167. 
Botsford, L., 1986. Effects of environmental forcing on age-structured populations: northern California Dungeness crab (Cancer magister) as an example. Canadian Journal of Fisheries and Aquatic Sciences 43 (11), 2345-2352.

Botsford, L., 1997. Dynamics of populations with density-dependent recruitment and age structure. In: Tuljapurkar, S., Caswell, H. (Eds.), Structured Population Models in Marine, Terrestrial, and Freshwater Systems. Chapman and Hall, New York, Ch. 12.

Botsford, L., Lawrence, C., Hill, M., 2005. Differences in dynamic response of california current salmon species to changes in ocean conditions. Deep-Sea Research II (52), 331-345.

Botsford, L., Lawrence, C. A., 2002. Patterns of co-variability among california current chinook salmon, coho salmon, dungeness crab, and physical oceanographic conditions. Progress in Oceanography 53, 283-305.

Bradford, M., 1995. Comparative review of Pacific salmon survival rates. Canadian Journal of Fisheries and Aquatic Sciences 52, 1327-1338.

Caswell, H., 2001. Matrix population models: construction, analysis, and interpretation. Sinauer, Sunderland, MA.

Coronado, C., Hilborn, R., 1998. Spatial and temporal factors affecting survival in coho salmon (Oncorhynchus kisutch) in the Pacific Northwest. Canadian Journal of Fisheries and Aquatic Sciences 55 (9), 2067-2077.

Day, T., Rowe, L., 2002. Developmental thresholds and the evolution of reaction norms for age and size at life history transitions. American Naturalist 159, $338-350$.

Doornik, D. M., Ford, M. J., Teel, D. J., 2002. Patterns of temporal genetic variation in coho salmon: estimates of the effective proportion of 2-year-olds in natural and hatchery populations. Transactions of the American Fisheries Society 131, 1007-1019. 
Elaydi, S. N., 1999. An Introduction to Difference Equations, 2nd Edition. Undergraduate Texts in Mathematics. Springer, New York.

Greenman, J. V., Benton, T. G., 2003. The amplification of environmental noise in population models: causes and consequences. The American Naturalist 161 (2), 225-239.

Greenman, J. V., Benton, T. G., 2005. The frequency spectrum of structured discrete time population models: its properties and their ecological implications. Oikos 110, 369-389.

Healey, M., 1991. Life history of Chinook salmon (Oncorhynchus tshawytscha). In: Groot, C., Margolis, L. (Eds.), Pacific Salmon Life Histories. UBC Press, Vancouver, Canada, pp. 313-393, 564 pp.

Hill, M., Botsford, L., Hastings, A., 2003. The effects of spawning age distribution on salmon persistence in fluctuating environments. Journal of Animal Ecology 72, 732-744.

Hirsch, M. W., Smale, S., 1974. Differential Equations, Dynamical Systems, and Linear Algebra. Academic Press.

Hsieh, C., Reiss, C., Hunter, J., Beddington, J., May, R., Sugihara, G., 2006. Fishing elevates variability in the abundance of exploited species. Nature 443 (7113), 859-862.

Jones, P., Briffa, K., Osborn, T., Lough, J., van Ommen, T., Vinther, B., Luterbacher, J., Wahl, E., Zwiers, F., Mann, M., Schmidt, G., Ammann, C., Buckley, B., Cobb, K., Esper, J., Goosse, H., Graham, N., Jansen, E., Kiefer, T., Kull, C., Kuttel, M., Mosley-Thompson, E., Overpeck, J., Riedwyl, N., Schulz, M., Tudhope, A., Villalba, R., Wanner, H., Wolff, E., Xoplaki, E., 2009. High-resolution palaeoclimatology of the last millennium: a review of current status and future prospects. The Holocene 19 (1), 3-49.

Logerwell, E., Mantua, N., Lawson, P., Francis, R., Agostini, V., 2003. Tracking environmental processes in the coastal zone for understanding and predicting 
Oregon coho (Oncorhynchus kisutch) marine survival. Fisheries Oceanography $12(6), 554-568$.

McCann, K., Botsford, L., Hastings, A., 2003. Differential response of marine populations to climate forcing. Canadian Journal of Fisheries and Aquatic Sciences 60 (8), 971-985.

Moloney, C. L., Botsford, L., Largier, J. L., 1994. Development, survival and timing of metamorphosis of planktonic larvae in a variable environment: the dungeness crab as an example. Marine Ecology Progress Series 113, 61-79.

Myers, R. A., Mertz, G., Bridson, J. M., Bradford, M. J., 1998. Simple dynamics underlie sockeye salmon (Oncorhynchus nerka) cycles. Canadian Journal of Fisheries and Aquatic Sciences 55, 2355-2364.

Nisbet, R. M., Gurney, W. S. C., 1982. Modelling Fluctuating Populations. Wiley, Chichester.

Perry, R. I., Cury, P., Brander, K., Jennings, S., Mllmann, C., Planque, B., 2010. Sensitivity of marine systems to climate and fishing: concepts, issues and management responses. Journal of Marine Systems 79, 427-435.

Pyper, B., Peterman, R., Lapointe, M., Walters, C., 1999. Patterns of covariation in length and age at maturity of British Columbia and Alaska sockeye salmon (Oncorhynchus nerka) stocks. Canadian Journal of Fisheries and Aquatic Sciences 56, 1046-1057.

Ricker, W., 1954. Stock and Recruitment. Fisheries Research Board of Canada. Sandercock, F., 1991. Life history of coho salmon (Oncorhynchus kisutch). In: Groot, C., Margolis, L. (Eds.), Pacific Salmon Life Histories. UBC Press, Vancouver, Canada, pp. 397-445, 564 pp.

Sissenwine, M. P., Shepherd, J. G., 1987. An alternative perspective on recruitment overfishing and biological reference points. Canadian Journal of Fisheries and Aquatic Sciences 44, 913-918. 
Sykes, Z. M., 1969. On discrete stable population theory. Biometrics 25 (2), 285-293.

Teo, S., Botsford, L., Hastings, A., 2009. Spatio-temporal covariability in coho salmon (Oncorhynchus kisutch) survival, from California to southeast Alaska. Deep-Sea Research Part II.

Timmermann, A., Oberhuber, J., Bacher, A., Esch, M., Latif, M., Roeckner, E., 1999. Increased El Niño frequency in a climate model forced by future greenhouse warming. Nature 398, 694-697.

Vollestad, L., Peterson, J., Quinn, T., 2004. Effects of freshwater and marine growth rates on early maturity in male coho and chinook salmon. Transactions of the American Fisheries Society 133, 495-503.

Young, K., 1999. Environmental correlates of male life history variation coho salmon populations from two Oregon coastal basins. Transactions of the American Fisheries Society 128, 1-16. 


\title{
Appendix A. General Mathematical Results
}

\author{
The linearization of a stochastic map
}

$$
\vec{x}(t)=F(\vec{x}(t-1), \xi(t), \xi(t-1), \ldots, \xi(t-L)), \quad \vec{x}(t) \in \mathbb{R}^{n}, \quad \xi(t) \in \mathbb{R}
$$

where (without loss of generality) $\mathrm{E}(\xi(t))=0$, is

$$
\vec{y}(t) \approx \mathbf{J} \vec{y}(t-1)+\sum_{l=0}^{L} \vec{H}(l) \xi(t-l)
$$

where $\vec{x}(t)=\tilde{\vec{x}}+\vec{y}(t), \tilde{\vec{x}}$ is a fixed point of $F(\vec{x}, 0), \mathbf{J}=\left.\left(\frac{\partial F_{i}}{\partial x_{j}}\right)\right|_{(\tilde{\vec{x}}, 0)}$ is the Jacobian matrix of $F$ at $\vec{x}=\tilde{\vec{x}}$ and $\xi=0, \vec{H}(l)=\frac{\partial F}{\partial \xi(t-l)}(\tilde{\vec{x}}, 0)$ is a vector expressing the dependence of $F$ on the noise terms, and $L$ is the maximum time lag at which stochastic perturbations affect $F$ directly. Let $\vec{v}_{i}$ and $\vec{u}_{i}$ be the left and right eigenvectors of $\mathbf{J}$, respectively, and $\lambda_{i}$ its eigenvalues, so that $\vec{v}_{i} \mathbf{J}=\lambda_{i} \vec{v}_{i}$ and $\mathbf{J} \vec{u}_{i}=\lambda_{i} \vec{u}_{i}$. In this paper we only consider matrices that have all distinct eigenvalues.

We change to the natural coordinate system of $\mathbf{J}$ : Let

$$
\mathbf{U}=\left(\vec{u}_{1}, \ldots, \vec{u}_{n}\right), \mathbf{V}=\left(\begin{array}{c}
\vec{v}_{1} \\
\vdots \\
\vec{v}_{n}
\end{array}\right), \mathbf{\Lambda}=\left(\begin{array}{cccc}
\lambda_{1} & 0 & \ldots & 0 \\
0 & & \\
\vdots & \ddots & \vdots \\
& & & 0 \\
0 & \ldots & 0 & \lambda_{n}
\end{array}\right) \text {, }
$$

with $\mathbf{J}=\mathbf{U} \boldsymbol{\Lambda} \mathbf{V}=\sum_{i} \lambda_{i} \vec{u}_{i} \vec{v}_{i}$ and $\mathbf{U V}=\mathbf{V U}=\mathbf{I}$. Then for $\vec{w}=\mathbf{V} \vec{y}$,

$$
\begin{aligned}
\vec{w}(t)=\mathbf{V} \vec{y}(t) & =\mathbf{\Lambda} \vec{y}(t-1)+\sum_{l=0}^{L} \vec{G}(l) \xi(t-l) \\
& =\Lambda \vec{w}(t-1)+\sum_{l=0}^{L} \vec{G}(l) \xi(t-l)
\end{aligned}
$$

with $\vec{G}(l)=\mathbf{V} \vec{H}(l)$. Using the terms of this transformed vector $\vec{w}$, the vector of deviations from equilibrium can be written as a sum of eigenvectors, $\vec{y}=$ $\sum_{i} w_{i} \vec{u}_{i}$. Since $\boldsymbol{\Lambda}$ is diagonal, the dynamics of each $w_{i}(t)$ is uncoupled from the 
others:

$$
w_{i}(t)=\lambda_{i} w_{i}(t-1)+\sum_{l=0}^{L} g_{i}(l) \xi(t-l)
$$

where $g_{i}(l)=\vec{v}_{i} \vec{H}(l)$ is the $i$ th entry of $\vec{G}(l)$. Thus, $\vec{w}(t)$ is the state of the linear system decomposed into its independent subsystems, and the vectors $\vec{G}(l)$ represent the stochastic forcing resolved into the decomposed coordinate system.

\section{Forcing and measurement}

The entries of the transformed vector $\vec{G}$ reveal how strongly the environmental forcing acts on each subsystem of the linearized system. Different kinds of forcing (i.e. survival, age of maturation) are distributed differently among the different subsystems, characterized by the projection of the forcing into each eigenspace, $g_{i}(l)=\vec{v}_{i} \vec{H}(l)$ for each $i$ and $l$. If $g_{i}(l)=0$ for all $l$, there is no fluctuation in the subspace containing eigenvector $\vec{u}_{i}$, that is, no fluctuation in $w_{i}$. In general, the more $\vec{H}$ is aligned with certain eigenvectors $\vec{u}_{i}$, the more the fluctuations caused by the forcing signal will be concentrated in those subsystems.

Similarly, a particular measurement generally observes some subsystems more than others. Assume we are observing the population via a scalar measurement, whether annual total population, recruitment or catch, represented as $M(t)=Q(\vec{x}(t))$. Let us assume that $Q(\vec{x})$ is differentiable at the fixed point $\tilde{\vec{x}}$. In the weak-noise limit this quantity also can be described by a linear approximation,

$$
\begin{aligned}
Q(\vec{x}(t)) & =Q(\tilde{\vec{x}})+\sum_{i} \frac{\partial Q}{\partial x_{i}}(\tilde{\vec{x}}) y_{i}(t)+\mathrm{O}\left(|y|^{2}\right) \\
& =Q(\tilde{\vec{x}})+\sum_{i} q_{i} y_{i}(t)+\mathrm{O}\left(|y|^{2}\right) .
\end{aligned}
$$

45 The measurement $M(t)$ is approximated by the linear quantity $Q(\tilde{\vec{x}})+\sum_{i} q_{i} y_{i}(t)$, which differs by only a constant from $\sum_{i} q_{i} x_{i}(t)$, and fluctuation in either quantity has the same characteristics as fluctuation in $\sum_{i} q_{i} y_{i}(t)$. We can represent the linearized measurement as a vector product $\vec{q} \vec{y}(t)$, using a row vector 
$\vec{q}=\left(q_{1}, \ldots, q_{n}\right)$. Changing to the natural coordinates of $\mathbf{J}$,

$$
\vec{q} \vec{y}(t)=\vec{q} \sum_{i} w_{i}(t) \vec{u}_{i}=\sum_{i} \vec{q} \vec{u}_{i} w_{i}(t)=\sum_{i} m_{i} w_{i}(t) .
$$

650

651

$657 \quad$ or

$$
\hat{w}(z)=\left(1-\boldsymbol{\Lambda} z^{-1}\right)^{-1} \sum_{l=0}^{L} \vec{G}(l) z^{-l} \hat{\xi}(z) .
$$

${ }_{658}\left(1-\boldsymbol{\Lambda} z^{-1}\right)^{-1}$ is a diagonal matrix with entries $\frac{1}{1-\lambda_{i} z^{-1}}=\frac{z}{z-\lambda_{i}}$. Consequently 659 the $Z$-transformed deviation vector,

$$
\hat{y}(z)=\mathbf{U} \hat{w}(z)=\sum_{i=1}^{n}\left(\vec{u}_{i} \frac{z}{z-\lambda_{i}} \sum_{l=0}^{L} g_{i}(l) z^{-l} \hat{\xi}(z)\right),
$$

660 is a linear sum of rational functions of $z$, with peaks tending to be near the ${ }_{661}$ eigenvalues of $\mathbf{J}$. 
Similarly, in the frequency analysis of a measurement $M(t)$,

$$
\begin{aligned}
\hat{M}(z) & =\sum_{k=0}^{\infty} M(k) z^{-k} \\
& \approx \sum_{k}\left(Q(\tilde{\vec{x}})+\sum_{i} m_{i} w_{i}(k)\right) z^{-k} \\
& =\sum_{k} Q(\tilde{\vec{x}}) z^{-k}+\sum_{i} m_{i} \hat{w}(z) .
\end{aligned}
$$

${ }_{663}$ It is appropriate to discard the constant part of $M(t)$ since we are concerned 664 with year to year variation:

$$
\begin{aligned}
\sum_{i} m_{i} \hat{w}(z) & =\sum_{i}\left[m_{i} \sum_{l=0}^{L} g_{i}(l) \frac{z}{z-\lambda_{i}} z^{-l} \hat{\xi}(z)\right] \\
& =T_{M}(z) \hat{\xi}(z) .
\end{aligned}
$$

665 The frequency response $T_{M}(z)$ of the measurement $M(t)$ is a weighted sum of 666 the frequency responses for each subsystem, $T_{i}(z)=\frac{z}{z-\lambda_{i}}$, each weighted both ${ }_{667}$ by the strength of environmental forcing in that subsystem at each lag, $g_{i}(l)$, ${ }_{668}$ and by the "emphasis" of the subsystem in the measurement, $m_{i}$. 